\title{
Research on the Omnidirectional Interception Guidance Law for High Speed Maneuvering Targets \\ Guoyu Bai* ${ }^{* 1}$ Huairong Shen ${ }^{2}$ Yongsheng Duan ${ }^{3}$ Hongyi Tan $^{4}$ \\ 1,2,3 Department of Spaceflight Equipment, Equipment Academy, Beijing, China \\ ${ }^{4}$ Jiuquan Satellite Launch Centre, Jiuquan, Gansu, China
}

Keywords: Omni-Directional, Guidance Law, Maneuver, High-Speed, Target, Interception

\begin{abstract}
Aiming to intercept high-speed maneuvering targets, nonlinear model was established and relative dynamic equations between the interceptor and the target were analyzed. An omni-directional true proportional navigation (OTPN) guidance law was developed. In high-speed target interception, OTPN guidance law has a wide capture region and can automatically shift interception mode. Formulary analysis proved that target maneuver has no effect on the expression form of OTPN guidance law. Without any additional conditions, OTPN guidance law can meet the requirement to intercepting high-speed maneuvering targets. Numerical simulation results showed that OTPN guidance law can effectively intercept high-speed maneuvering targets and its capture region is influenced by targets' maneuver ability and initial LOS angle. The parameters of OTPN, such as capture region, ballistic characteristics, and overload variation and control efforts, show that OTPN are superior to traditional guidance schemes.
\end{abstract}

\section{Introduction}

Interception for maneuvering targets with high-speed is a formidable challenge, while the relative dynamic model between interceptor and target is a complicated and nonlinear model. Classical and modern guidance law both have difficulty in meeting the interception requirements of maneuvering target with high speed ${ }^{[1]}$, therefore, more effective guidance law should work out.

In present studies of guidance for intercepting high-speed target, Yuping $\mathrm{Li}^{[2]}$ etal. proposed a modified proportional navigation guidance law(MPNG) to intercept high speed target; Golan ${ }^{[3]}$ etal. developed a head-pursuit guidance law predicts the position of the target though time variable heading angle; Shima ${ }^{[4]}$ used sliding mode guidance method to intercept maneuvering target with impact angle constrains. Prasanna ${ }^{[5]}$ proposed a guidance law named retro-proportional navigation(RPN), which uses a negative navigation coefficient to intercept high-speed targets in the case of tail-chase engagement. Chaoyong $\mathrm{Li}^{[6]}$ etal. analyzed necessary initial and the singular conditions of interception for high speed target, and proposed a new guidance law based on differential geometry;JunhuiXiong ${ }^{[7]}$ etal. applied fuzzy variable coefficient in variable structure guidance law, though re- 
ducing earlier overload requirement to resolve the problem of intercepting target with high speed in head-on engagement; Liang Yan ${ }^{[8]}$ combined positive and negative proportional guidance into a united proportional guidance law, which can effectively improve capture region to the target with high speed.

Most researches on the guidance law for intercepting maneuvering targets, typically used augmented proportional navigation methodology, differential games methodology, and sliding-mode variable structure methodology. Tyan[9] designed a 3D pure proportional guidance law and derived its capture region; Der[10] presented a guidance law which called augmented proportional navigation guidance (APN), for intercepting hypersonic targets. Liying Yuan[11] applied fuzzy control into designing of guidance scheme, transformed guidance problem into an error-feedback control problem, and proposed an adaptive feedback linearization guidance law; Tian[12] etal. divided interception process into different periods based on the idea of suppression the angular rate of LOS, though which the pursuer could intercepting the target in approximated parallel approach trajectory; Jianliang Peng[13] presented a simple and fast non-oscillatory tracking differentiator, and used it in augmented proportion navigation; Kebo Li[14]proposed a new differential geometry guidance with geometric construction method of the acceleration, in which the target acceleration information can be ignored,and the form of control commands were simplified effectively; Humin Lei[15] investigated a robust guidance law based on flexible terminal sliding mode control theory, in which effectively eliminate the chatter of interception; Yuan Li[16] introduced a novel 3D united proportional navigation guidance for maneuvering target with high speed, which combines the advantages both of the head-on and head-pursuit engagement though time-varying navigation gain, which enlarged the capture region significantly.

To intercept the maneuvering target with high speed, three difficult problems should be solved: 1) because the target velocity is faster than that of the pursuer, tail-chaseinterception became unrealized, only head-on and head-pursuit interception be realizable; 2) to intercept a maneuvering target, sliding mode variable structure theory takes the target's maneuvering as system errors to overcome, which makes the process of interception been chattering near the sliding surface; 3 ) the augmented proportional navigation guidance and differential game guidance law must be based on the assuming values of the parameters about target maneuvering, which in actual interception was unrealistic.

To solve the problem of interception for maneuvering target with high speed, this paper applied TPN basic setting and thoughts [17 20] as a starting point of research and considered the interception's characteristics in the outer atmosphere. Firstly, the nonlinear model was established and the relative dynamic equations between the interceptor and the target with high speed were analyzed.Aomni-directional true proportional navigation guidance(OTPN) law has been developed, which has significantly wide capture region for the high speed target.The guidance law is adapted for variable-speed interception and can auto- 
matically shift interception mode, possessing both head-pursuit and head-on interception capabilities. Furthermore, the maneuvering parameters of target were considered in the dynamic equations, though we figured out that the OTPN guidance law can satisfy the interception requirement of maneuvering target without adding any correction terms. The results of numerical simulation confirmed the viability and validity of OTPN guidance law for maneuvering target interception, which can achieve a wider capture region than the contrast guidance schemes. In terms of the parameter of coverage, ballistic characteristics, acceleration, total control efforts and comprehensive evaluation, OTPN guidance law had obvious advantages than the contrast guidance methods.

\section{Problem Formulation}

Vector Analysis of Engagement in 3D Space

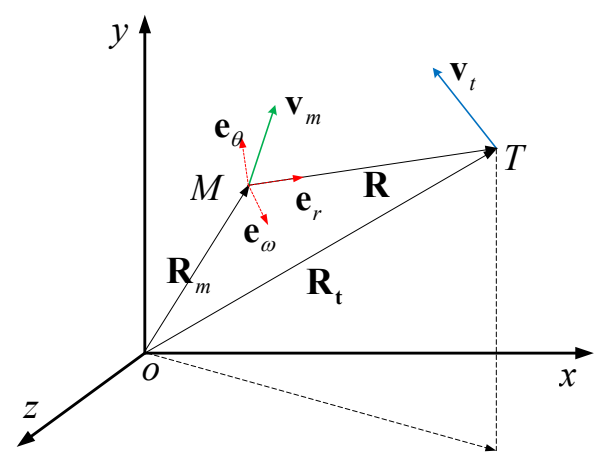

Figure 1. Relative motion of vectors in $3 \mathrm{D}$ space

In order to conveniently analyze the relative motion between interceptor and target, the interceptor and target is regarded as particles in 3D space, neglecting the influence of gravity. The geometry of the engagement between target and pursuer in $3 \mathrm{D}$ space is presented in Figure 1.The position of interceptor is point $M$, and target located on point $T$; the interceptor's position vector is $\mathbf{R}_{m}$, position vector of the target is $\mathbf{R}_{t}$, interceptor's velocity vector is $\mathbf{V}_{m}$, and the target velocity vector is $\mathbf{V}_{t}$.As shown in Figure 1, the relative position vector of interceptor to target is:

$$
\mathbf{R}=\mathbf{R}_{t}-\mathbf{R}_{m}
$$

The rotation coordinate system of LOS is defined as $M(r, \omega, \theta)$, whose coordinate origin point at $M, \mathbf{e}_{r}$ vector point to target $T$. The rotation of the LOS in three dimensional space can be deconstructed into the rotation of LOS in the instant rotation plane and the rotation of instant rotation plane, the instantaneous rotation plane defined by $\left(\mathbf{e}_{r}, \mathbf{e}_{\theta}\right)$, the rotation rate of LOS in instantaneous rotation plane is $\omega_{s}$, and rotating axis is $\mathbf{e}_{\omega}$, where:

$$
\boldsymbol{\omega}_{s}=\omega_{s} \mathbf{e}_{\omega}
$$


The rotating axis of instantaneous rotation plane is $\mathbf{e}_{r}$, and $\boldsymbol{\Omega}_{s}$ is defined as the rate of rotation, which:

$$
\mathbf{\Omega}_{s}=\Omega_{s} \mathbf{e}_{r}
$$

In LOS rotation coordinate system, we have:

$$
\left\{\begin{array}{l}
\frac{d \mathbf{e}_{r}}{d t}=\omega_{s} \mathbf{e}_{\theta} \\
\frac{d \mathbf{e}_{\theta}}{d t}=-\omega_{s} \mathbf{e}_{r}+\Omega_{s} \mathbf{e}_{\omega} \\
\frac{d \mathbf{e}_{\omega}}{d t}=-\Omega_{s} \mathbf{e}_{\theta}
\end{array}\right.
$$

Deconstruct the accelerations of target and interceptor in the LOS rotation coordinate system:

$$
\left\{\begin{array}{l}
a_{t r}-a_{m r}=\ddot{R}-R \omega_{s}^{2} \\
a_{t \theta}-a_{m \theta}=2 \dot{R} \omega_{s}+R \dot{\omega}_{s} \\
a_{t \omega}-a_{m \omega}=\mathrm{R} \omega_{s} \Omega_{s}
\end{array}\right.
$$

Planar guidance laws in instantaneous rotation plane can be constructed to serve for $3 \mathrm{D}$ interception guidance ${ }^{[20]}$, thus in this paper, only the planar attack is considered. In instantaneous rotation plane, we get:

$$
\left\{\begin{array}{l}
a_{t r}-a_{m r}=\ddot{R}-R \omega_{s}^{2} \\
a_{t \theta}-a_{m \theta}=2 \dot{R} \omega_{s}+R \dot{\omega}_{s}
\end{array}\right.
$$

Planar Relative Dynamic Equation. In this section we consider the dynamic formulation of the planar intercept problem used in the derivation of the guidance law. The dynamic relation between the interceptor and target in 2-dimensional plane is depicted in Figure 2.

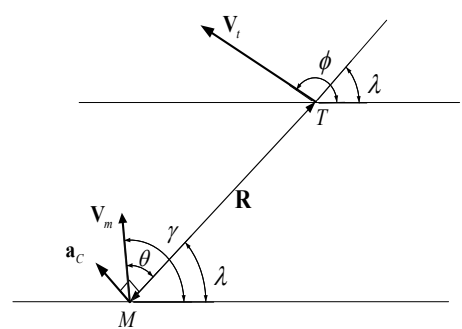

Figure 2. Relative motion between interceptor and target

In Figure 2, the relative position vector of interceptor to target is $\mathbf{R}, \mathbf{V}_{t}$ is the velocity vector of target, $\mathbf{V}_{m}$ is the velocity vector of interceptor, ${ }^{\phi}$ is the angle between velocity of target and the reference line, $\lambda$ is the angle between the LOS and reference line, $\gamma$ is the interceptor's flight-path angle, the angle between $\mathbf{V}_{m}$ and LOS de- 
fined as heading angle $\theta$,and $\mathbf{a}_{C}$ is the control acceleration of the interceptor and perpendicular to the LOS direction. From Figure 2, it is convenient to obtain the relative dynamic equations between the pursuer and target.

$$
\begin{aligned}
& R \dot{\lambda}=V_{t} \sin \left(\phi^{-} \lambda\right)-V_{m} \sin (\gamma-\lambda) \\
& \dot{R}=V_{t} \cos \left(\phi^{-} \lambda\right)-V_{m} \cos (\gamma-\lambda)
\end{aligned}
$$

Note that the relationship between $\gamma$ and $\lambda$ in proportional navigation:

$$
\dot{\gamma}=N \dot{\lambda}
$$

whereNis navigation gain. For:

$$
\theta=\gamma-\lambda
$$

We have:

$$
\dot{\theta}=\dot{\gamma}-\dot{\lambda}=N \dot{\lambda}-\dot{\lambda}=(N-1) \dot{\lambda}
$$

Engagement Kinematics. Due to the fact that the control acceleration and the velocity of the interceptor is not orthogonal; the acceleration $\mathbf{a}_{C}$ will affects the velocity value, which leads $V_{m}$ to a variable value. The acceleration $a_{C}$ in the interceptor's velocity coordinate system is decomposed into component of the magnitude of the interceptor's velocity ${ }^{a_{V}\left(\dot{V}_{m}\right)}$ and the component of the velocity direction $a_{\perp V}\left(V_{m} \dot{\gamma}\right)$.

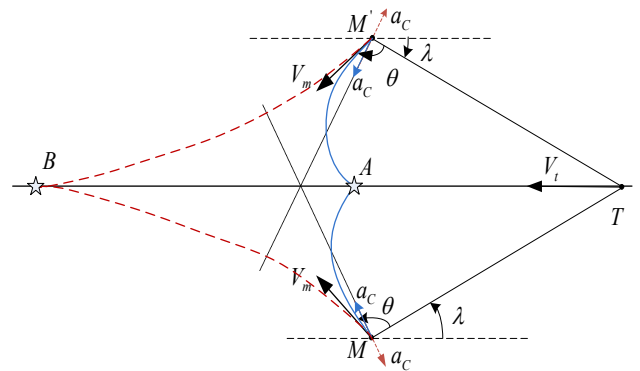

Figure 3. Guidance by PN on velocity head angle $\theta$

Figure 3 shows that beginning with same initial head angle, the counter direction of command acceleration makes the interceptor's velocity direction change to the opposite trend as well as the value of the velocity, and the intercepting trajectory is also different too.

When the PN guidance law guides the interceptor achieve at $\dot{\lambda}=0$ or successfully engaged the target as $R_{f} \longrightarrow 0$, in these two cases, the 2nd formula of Equation(7) becomes:

$$
V_{t f} \sin \left(\phi_{f}-\lambda_{f}\right)=V_{m f} \sin \left(\gamma_{f}-\lambda_{f}\right)
$$


The flight path angle of the interceptor at engage moment:

$$
\gamma_{f}=\lambda_{f}+n \pi+(-1)^{n} \arcsin \left(\beta_{f} \sin \left(\phi_{f}-\lambda_{f}\right)\right)
$$

Where $\beta_{f}=V_{t f} / V_{m f}$ is the ratio of velocity at collision, accounting the physical meaning and the value of $\gamma$ and $\lambda, n$ in Equation (12) should be 0 or 1, thus:

$$
\begin{aligned}
& \gamma_{f 1}=\lambda_{f}+\arcsin \left(\beta_{f} \sin \left(\phi_{f}-\lambda_{f}\right)\right) \\
& \gamma_{f 2}=\lambda_{f}+\pi-\arcsin \left(\beta_{f} \sin \left(\phi_{f}-\lambda_{f}\right)\right)
\end{aligned}
$$

From Equation (13), it is clear that: for the fixed target flight path angle ${ }^{\phi_{f}}$, the LOS angle $\lambda_{f}$ and the final velocity ratio $\beta_{f}$, there are two different final path angles $\gamma_{f 1}$ and $^{\gamma_{f 2}}$ for interceptor to engage with the target successfully. When being guided by the PN guidance, the interceptor's path angle is towards to ${ }^{\gamma_{f 1}}$, and the navigation gain is positive, which will be negative when guided to the terminal path angle $\gamma_{f 2}$.

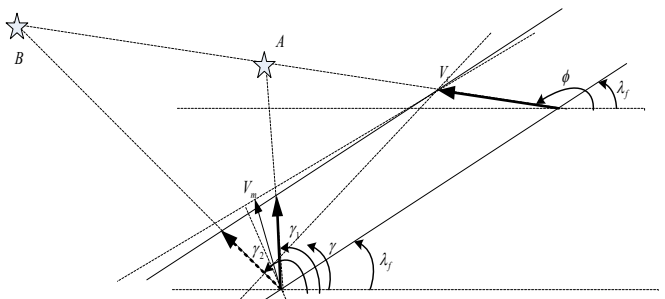

Figure 4. Geometry of interceptor and target engaged successfully in different final path angle

Be constrained with the target path angle ${ }^{\phi}$, the velocity $\operatorname{ratio} \beta$, and the LOS angle $\lambda$, interceptor engaged toward target on two different terminal path angles. The relationship between $\gamma_{1}$ and $\gamma_{2}$ is shown in Figure 4 and Equation (13):

$$
\left(\gamma_{1}-\lambda_{f}\right)+\left(\gamma_{2}-\lambda_{f}\right)=\theta_{1}+\theta_{2}=\pi
$$

The interceptor's initial heading angle $\theta$ defined in region $[-\pi, \pi]$ : while $|\theta|<\pi / 2$, the navigation gain $N$ takes positive; while $|\theta|>\pi / 2$, navigation gain takes positive but minus sign is added in the guidance equation, the Equation (8) becomes:

$$
\begin{cases}\frac{d \gamma}{d t}=N \frac{d \lambda}{d t} & \left(|\theta|<\frac{\pi}{2}\right) \\ \frac{d \gamma}{d t}=-N \frac{d \lambda}{d t} & \left(|\theta|>\frac{\pi}{2}\right)\end{cases}
$$

Guidance to Collision. Differentiating Equation (7):

$$
\begin{aligned}
\ddot{R}= & \dot{V}_{t} \cos (\phi-\lambda)-V_{t}(\dot{\phi}-\dot{\lambda}) \sin (\phi-\lambda) \\
& -\dot{V}_{m} \cos (\gamma-\lambda)+V_{m}(\dot{\gamma}-\dot{\lambda}) \sin (\gamma-\lambda)
\end{aligned}
$$


Substitute Equation (8) into Equation (16):

$$
\begin{aligned}
\ddot{R}= & \dot{V}_{t} \cos (\phi-\lambda)-V_{t} \dot{\phi} \sin (\phi-\lambda)-\dot{V}_{m} \cos (\gamma-\lambda) \\
& +\left[V_{t} \sin (\phi-\lambda)+(N-1) V_{m} \sin (\gamma-\lambda)\right] \dot{\lambda}
\end{aligned}
$$

Substituting Equation (7),we have:

$$
\begin{aligned}
\ddot{R}= & \dot{V}_{t} \cos (\phi-\lambda)-V_{t} \dot{\phi} \sin (\phi-\lambda)- \\
& \dot{V}_{m} \cos (\gamma-\lambda)+N V_{m} \dot{\lambda}_{\sin } \sin (\gamma)+R \dot{\lambda}^{2}
\end{aligned}
$$

The heading angle of target defined by ${ }^{\varphi=\phi-\lambda}$,Equation (18) becomes:

$$
\ddot{R}=\dot{V}_{t} \cos \varphi-V_{t} \dot{\phi} \sin \varphi-\dot{V}_{m} \cos \theta+N V_{m} \dot{\lambda} \sin \theta+R \dot{\lambda}^{2}
$$

Note that: ${ }^{\omega_{s}=\dot{\lambda}}$,Equation (6)becomes:

$$
\left\{\begin{array}{l}
a_{t r}-a_{m r}=\ddot{R}-R \dot{\lambda}^{2} \\
a_{t \theta}-a_{m \theta}=2 \dot{R} \dot{\lambda}+R \ddot{\lambda}
\end{array}\right.
$$

The interceptor's acceleration perpendicular to the LOS, in Equation (20), $a_{m r}=0$,thus:

$$
\ddot{R}-R \dot{\lambda}^{2}=a_{t r}
$$

Substitute Equation (21)into Equation (18):

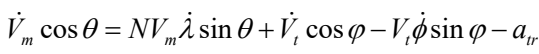

Assuming that the target velocity is constant and there is no avoiding behavior, inEquation (22), ${ }_{V_{t}}=0, \dot{\phi}=0, a_{t r}=0$, therefore:

$$
\dot{V}_{m}=N V_{m} \frac{\sin (\gamma-\lambda)}{\cos (\gamma-\lambda)} \dot{\lambda}=N V_{m} \tan (\gamma-\lambda) \dot{\lambda}=N V_{m} \tan \theta \dot{\lambda}
$$

The control acceleration of interceptor is:

$$
a_{C}=\frac{\dot{V}_{m}}{\sin \theta}=\frac{N V_{m}}{\cos (\gamma-\lambda)} \dot{\lambda}
$$

Combined Equation (24) and Equation (15), a novel guidance law which can intercept target both in head-on and head-pursuit mode was proposed, which required looser initial conditions than other guidance schemes; this new guidance law can guide the interceptor under head-on or head-pursuit engagement mode, thus the capture region and the efficiency of interception were enhanced significantly. The new guidance law can intercept the target on any initial path angle, and automatically alter the sign of proportional coefficient to achieve interception guidance in all directions. A new guidance law was named as Omni directional True Proportion Navigation guidance law(OTPN), and the guidance control equation of OTPN is: 


$$
\left\{\begin{array}{lr}
a_{C}=\min \left(\frac{N V_{m}}{\cos \theta} \dot{\lambda}, \operatorname{sgn}\left(\frac{N V_{m}}{\cos \theta} \dot{\lambda}\right)\left|a_{\max }\right|\right) & \left(|\theta|<\frac{\pi}{2}\right) \\
a_{C}=\min \left(\frac{-N V_{m}}{\cos \theta} \dot{\lambda}, \operatorname{sgn}\left(\frac{-N V_{m}}{\cos \theta} \dot{\lambda}\right) \mid a_{\max }\right)\left(|\theta|>\frac{\pi}{2}\right) & \left(\theta= \pm \frac{\pi}{2}\right) \\
a_{C}=0 & (\theta)
\end{array}\right.
$$

\section{OTPN Guidance of Interception for Maneuvering Target with High Speed}

In case of the target maneuvering, from Equation (22), we know that:

$$
\begin{gathered}
\dot{V}_{m}=\frac{N V_{m} \sin \theta+\dot{V}_{t} \cos \varphi-V_{t} \dot{\phi} \sin \varphi-a_{t r}}{\cos \theta} \\
\dot{V}_{m}=N V_{m} \dot{\lambda} \tan \theta+\frac{\cos \varphi}{\cos \theta} \dot{V}_{t}-\frac{\sin \varphi}{\cos \theta} V_{t} \dot{\phi}-\frac{a_{t r}}{\cos \theta}
\end{gathered}
$$

The guidance control acceleration becomes that:

$$
\begin{aligned}
a_{C}= & \frac{\dot{V}_{m}}{\sin \theta}=\frac{N V_{m} \dot{\lambda}}{\cos \theta}+\frac{\cos \varphi}{\sin \theta \cos \theta} \dot{V}_{t} \\
& -\frac{\sin \varphi}{\sin \theta \cos \theta} V_{t} \dot{\phi}-\frac{a_{t r}}{\sin \theta \cos \theta}
\end{aligned}
$$

For maneuvering target with high speed, the ratio between the angular rate of flight path and the LOS angle should be kept constant, and the command acceleration should satisfyEquation (28). Decompose the acceleration of maneuvering target in velocity coordinate system and LOS coordinate system, as shown in Figure 5 :

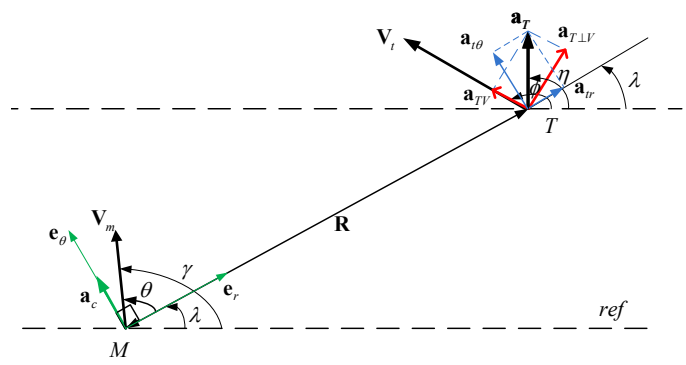

Figure 5. Target acceleration in the velocity coordinate system and the LOS coordinate system

In Figure 5, where target acceleration is $a_{T}$, the angle between the reference line and $a_{T}$ defined as $\eta$, decomposing $a_{T}$ in the velocity coordinate system of target:

$$
\begin{aligned}
& a_{T V}=a_{T} \cos (\phi-\eta)=\dot{V}_{t} \\
& a_{T \perp V}=a_{T} \sin (\phi-\eta)=-V_{t} \dot{\phi}
\end{aligned}
$$

Decompose $a_{T}$ in the LOS coordinate system: 


$$
\begin{aligned}
& a_{t r}=a_{T} \cos (\eta-\lambda) \\
& a_{t \theta}=a_{T} \sin (\eta-\lambda)
\end{aligned}
$$

Substitute Equation (29)-(32)into Equation (27):

$$
\begin{aligned}
\dot{V}_{m} \cos \theta= & N V_{m} \dot{\lambda} \sin \theta+a_{T} \cos (\phi-\eta) \cos (\phi-\lambda) \\
& +a_{T} \sin (\phi-\eta) \sin (\phi-\lambda)-a_{T} \cos (\eta-\lambda)
\end{aligned}
$$

Simplify Equation (33):

$$
\dot{V}_{m} \cos \theta=N V_{m} \dot{\lambda} \sin \theta
$$

So the command acceleration still is:

$$
a_{C}=\frac{N V_{m} \dot{\lambda}}{\cos \theta}
$$

According to the formula deriving, the change effect of amplitude and direction is included in the angular rate of the LOS, and the maneuvering of the target has no effect on the expression form of OTPN guidance law. Without any additional items, the OTPN guidance law can meet the requirement of intercepting maneuvering target with high speed.

\section{Simulation and Discussion}

The scenarios presented in this section are variant of the same configuration, in which initial distance between pursuer and target is $R_{0}=10000 \mathrm{~m}$, the initial LOS angle $\lambda_{0}=10^{\circ}$, target with velocity of $1500 \mathrm{~m} / \mathrm{s}$ and position with $\left(X_{t}\right.$, $\left.Y_{t}\right)=(10000 \mathrm{~m}, 10000 \mathrm{~m})$, and the initial path angle of target is $\phi_{0}=180^{\circ}$; the initial position of the pursuer can be calculated by $R_{0}$ and $\lambda_{0}$, initial velocity of pursuer defined as $V_{i}=600 \mathrm{~m} / \mathrm{s}$, and the initial path angle of interceptor being variable as $\gamma_{0}=0 \sim 360^{\circ}$, the navigation gain of all scenarios is $N=6$.

Simulations were run in MATLAB environment where they were terminated when $V_{c}<0$. The range value $R_{f}$ at final instant happens to be the miss distance. The seeker model is of a perfect seeker (without noise) with a sufficient large field of view, but it is assumed to be unable to track the target when the range is shorter than $30 \mathrm{~m}$, after which the guidance command maintains its last value. The effective interception occurred when the minimum distance between interceptor and target is less than $10 \mathrm{~m}$.

In the numerical simulation, the acceleration of target's maneuvering keeps constant, and it keeps perpendicular to the velocity direction of the target in the interception process. The acceleration is defined as:

$$
a_{t}=n * g \quad(n \in R)
$$


The Classic Proportional Navigation (Pn) Guidance Scenario. The proportional navigation $(\mathrm{PN})$ law states that the control acceleration of interceptor is proportional to the LOS rate:

$$
\frac{d \gamma}{d t}=N \frac{d \lambda}{d t}
$$

The acceleration of the target is valued respectively as $0,1,2$, and $5 \mathrm{~g}$, and PN guidance scenario for the initial path angle range of the effective interception of maneuvering target is shown in Table 1. Simulation results indicate that the interception fails when the maneuvering acceleration of target exceeds $9 \mathrm{~g}$.

Table 1.Simulation results using the $1 \sim 4$ guidance schemes to intercepting maneuvering target with high speed

\begin{tabular}{ccccccc}
\hline & & Maximum & \multicolumn{4}{c}{ Successful initial path angle } \\
\cline { 3 - 7 } Scenario & $\begin{array}{c}\text { Acceleration } \\
\text { of target }\end{array}$ & $\begin{array}{c}\text { of the inter- } \\
\text { ceptor's ac- } \\
\text { celeration }\end{array}$ & PN(deg) & APN(deg) & $\begin{array}{c}\text { RPN } \\
(\mathrm{deg})\end{array}$ & ARPN(deg) \\
\hline 1 & 0 & $30 \mathrm{~g}$ & $9^{\circ} \sim 77^{\circ}$ & $9^{\circ} \sim 77^{\circ}$ & $58^{\circ} \sim 213^{\circ}$ & $58^{\circ} \sim 213^{\circ}$ \\
2 & $1 \mathrm{~g}$ & $30 \mathrm{~g}$ & $18.5^{\circ} \sim 85^{\circ}$ & $16^{\circ} \sim 81.5^{\circ}$ & $55^{\circ} \sim 189^{\circ}$ & $56^{\circ} \sim 193^{\circ}$ \\
3 & $2 \mathrm{~g}$ & $30 \mathrm{~g}$ & $27.5^{\circ} \sim 92.5^{\circ}$ & $21.5^{\circ} \sim 85^{\circ}$ & $53^{\circ} \sim 164^{\circ}$ & $55^{\circ} \sim 173^{\circ}$ \\
4 & $5 \mathrm{~g}$ & $30 \mathrm{~g}$ & $57^{\circ} \sim 113^{\circ}$ & $45^{\circ} \sim 97.5^{\circ}$ & $51^{\circ} \sim 99^{\circ}$ & $55^{\circ} \sim 118^{\circ}$ \\
\hline
\end{tabular}

Augmented Proportional Navigation(APN) Guidance Scenario. Augmented proportional navigation based on the assuming of target's maneuvering acceleration is constant, and an additional square term $0.5 \mathrm{a}_{\mathrm{T}} \mathrm{t}_{\mathrm{go}}{ }^{2}$ to the zero-effort miss(ZEM) is converted into a basic variables the proportional guidance law ${ }^{[21]}$. In augmented proportional navigation(APN) guidance, the acceleration is:

$$
a_{C}=N V_{m}\left(\dot{\lambda}+0.5 a_{T}\right)
$$

The value of target's acceleration is completely same as the PN scenario and the simulation results of the augmented proportional navigation guidance is shown in Table 1, and the interception fails when the maneuvering acceleration of target exceeds $9 \mathrm{~g}$ also.

Retro-Proportional Navigation Guidance (RPN) Scenario. Retro-proportional guidance ${ }^{[7]}$ defines that navigation gain is negative to intercepting target in head-pursuit mode, and the guidance differential equation is:

$$
\frac{d \gamma}{d t}=-N \frac{d \lambda}{d t}
$$

The initial velocity angle range of the successful interception of RPN scheme is also shown in Table 1. When target's maneuvering acceleration is larger than $10 \mathrm{~g}$, the RPN guidance scheme fails to intercept. 
Augmented Retro-Proportional Navigation Guidance Scenario. Based on RPN guidance, adding correction term about target's acceleration will be beneficial to the intercepting affection for maneuvering target. The basic thought of augmented retro-proportional navigation is in completely agreement with APN. The control acceleration of interceptor is:

$$
a_{C}=-N V_{m}\left(\dot{\lambda}+0.5 a_{T}\right)
$$

Simulation results of the ARPN guidance are shown in Table 1, and ARPN can't finish the interception successfully while maneuvering acceleration of the target is greater than $9 \mathrm{~g}$.

OTPN Guidance Scenario. According to the initial head angle of interceptor, the proposed guidance law can switch automatically between head-on and head-pursuit interception, whose control acceleration equation is Equation (40). The simulation results are shown in Table 2 and Figure 6, which show that guided with the OTPN guidance law, the interceptor's capture region for maneuvering target with high speed were significantly enlarged. As the maximum acceleration of interceptor is $30 \mathrm{~g}$, even the target's maneuvering acceleration is increased to $25 \mathrm{~g}$, capture region still exists. The OTPN guidance law almost keeps the omnidirectional capture ability while maneuvering acceleration of target is small enough.

Table2.Simulation results using OTPN guidance law to intercept maneuvering target

\begin{tabular}{ccc}
\hline $\begin{array}{c}\text { Sce- } \\
\text { nario }\end{array}$ & $\begin{array}{c}\text { Accelera- } \\
\text { tion of } \\
\text { target }(\mathrm{g})\end{array}$ & $\begin{array}{c}\text { Successful ini- } \\
\text { tial path angle } \\
\text { of OTPN(deg) }\end{array}$ \\
\hline 1 & 0 & $0^{\circ} \sim 280^{\circ}$, \\
$285^{\circ} \sim 360^{\circ}$ \\
$0^{\circ} \sim 280^{\circ}$, \\
$285^{\circ} \sim 360^{\circ}$ \\
$0^{\circ} \sim 277^{\circ}$, \\
2 & 1 & $285^{\circ} \sim 303^{\circ}$ \\
& & $24^{\circ} \sim 147^{\circ}$, \\
3 & 5 & $285^{\circ} \sim 301^{\circ}$ \\
& & $46^{\circ} \sim 95.5^{\circ}$ \\
$45^{\circ} \sim 71^{\circ}$
\end{tabular}



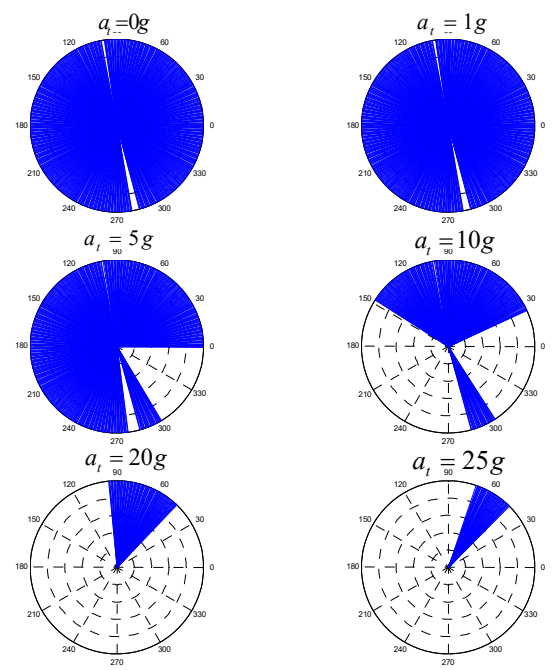

Figure 6. Capture region of OTPN for intercepting maneuvering targets with different acceleration $(0 \sim 25 \mathrm{~g})$

Figure 6 shows: 1) the capture region of OTPN guidance law is influenced by the acceleration ability of target; 2) the stronger the target's maneuvering abilityis, the smaller the capture range of OTPNis; 3) OTPN guidance law cannot achieve intercept target with stronger maneuver ability.

In order to study on the interception characteristics of OTPN in detail, in further simulations, the target's acceleration is fixed to constant of $10 \mathrm{~g}$, and value of the initial LOS angle of the interceptor is variable. The numerical simulation results are shown in Figure 7.
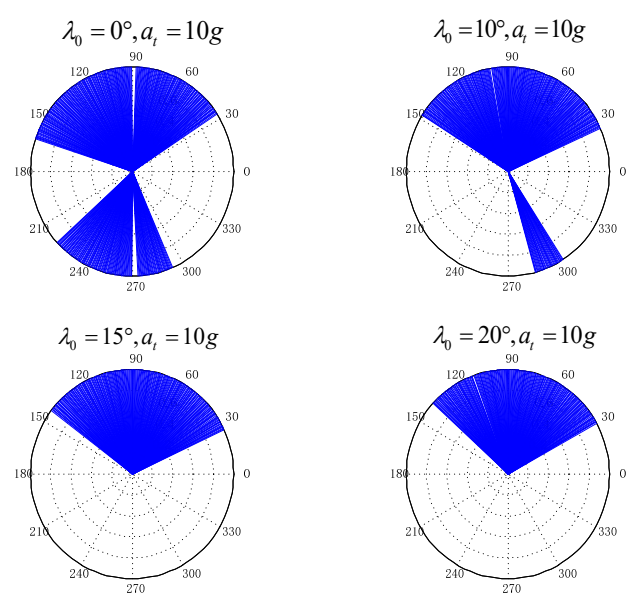

Figure 7. Capture region for intercepting maneuvering targets with different initial LOS angle by OTPN 

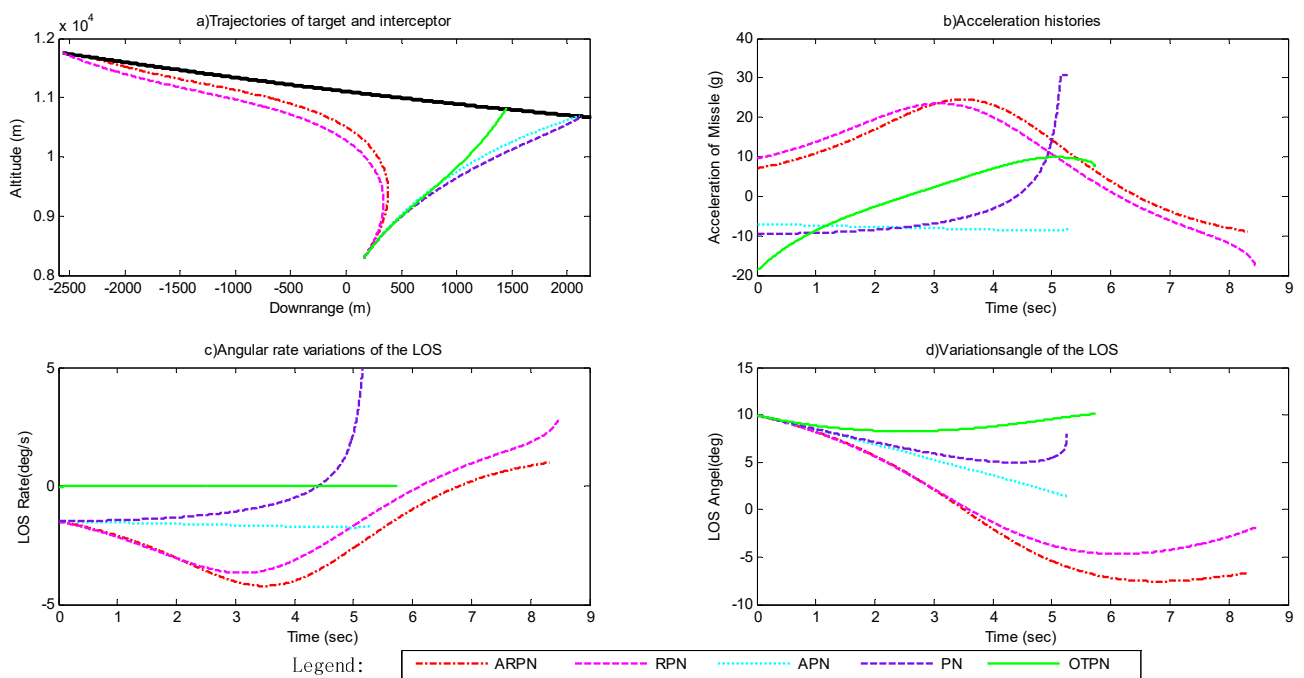

Figure 8. Simulation results of different guidance schemes underthe same initial conditions

Table 3. Simulation results of different guidance schemes under same initial conditions

\begin{tabular}{cccccc}
\hline Scheme & Miss distance $(\mathrm{m})$ & Maximum acceleration $(\mathrm{g})$ & terminal LOS angle $(\mathrm{deg})$ & Control efforts(m/s) & Total time $(\mathrm{s})$ \\
\hline ARPN & 0.1837 & 23.5165 & -1.8745 & 1103 & 8.4822 \\
RPN & 0.0993 & 24.5506 & -6.7002 & 1038 & 8.3262 \\
APN & 0.1047 & 8.7205 & 1.4046 & 412.1918 & 5.2886 \\
PN & 1.0706 & 30.0000 & 7.9534 & 412.1441 & 5.2726 \\
OTPN & 0.1520 & 19.0269 & 10.1180 & 396.0155 & 5.7380 \\
\hline
\end{tabular}

Comparison of Different Guidance Scenarios. Under same initial conditions, which initial LOS angle is $10^{\circ}$ and interceptor initial velocity flight path angle is $70^{\circ}$, maneuvering acceleration of target keeps constant of $5 \mathrm{~g}$. Guidance simulation results of ARPN, RPN, APN, PN and OTPN are shown in Figure 8 and Table 3.

Numerical simulation shows that: ARPN and RPN spent the longest total time consumed in interception, and their whole control efforts is the biggest too; acceleration requirement and miss distance of PN guidance law are bigger than others; trajectory of OTPN is the straightest, whose angular rate of LOS was more smoothly, and requirement of control effort is the minimal.Combined the advantages of OTPN in capture region and adaptability for target's maneuver, comparison of these schemes indicated that OTPN is superior to other schemes in performance of interception for maneuvering targets with high speed. 


\section{Conclusions}

This paper put forward a new guidance law called omnidirectional true proportional navigation (OTPN), which is developed for intercepting maneuvering targets with high-speed. OTPN can automatically alter interception mode, possessing both head-pursuit and head-on interception capabilities.Considered maneuvering parameters of target, though formulary deriving, this paper found that OTPN guidance equation iscompletely compatible to maneuveringof target without any modified terms. Using OTPN guidance law to intercept maneuvering target can effectively avoid parameters estimate error's affection on guidance accuracy. Therefore, OTPN not only has the omnidirectional interception ability to high speed target, but also can effectively deal with maneuver evasion target.

Numerical simulation results shows: OTPN can effectively accomplish the intercepting guidance to maneuvering target, whose capture region is influenced by the maneuvering ability of target and the initial LOS angle. Compared with classic PN, RPN, APN, and ARPN guidance schemes in same conditions, the parameters of OTPN guidance law such as capture region, ballistic characteristics, overload variation and control efforts are all superior to traditional guidance schemes.

\section{References}

[1] Siouris G M. 2004. Missile Guidance and Control Systems. New York:Springer, pp: 142-173.

[2] Yuping L, Chunliang L, Yunhao L.2013. Development of 3-D modified proportional navigation guidance law against high-speed targets. IEEE Transactions on Aerospace and Electronic Systems, 49(1): 677-687.

[3] Oded M. Golan, Tal Shima. 2004. Head pursuit guidance for hypervelocity interception. AIAA Guidance, Navigation, and Control Conference. Providence, RI(US), pp:1437-1444.

[4] Shima T. 2011. Intercept-angle guidance. Journal of Guidance, Control, and Dynamics, 34(2): 484-492.

[5] Prasanna H M, Ghose D. 2012. Retroproportionalnavigation: A new guidance law for interception of high speed targets. Journal of Guidance, Control, and Dynamics, 35(2): 377-386.

[6] LiChaoyong,JingWuxin,QiZiguo,et al.2007. Application of the 3D differential geometric guidance commands. Journal of Astronautics,28(5):1235-1240.

[7] XiongJunhui,Tang Shengjing,GuoJie, Zhu Dalin. 2014. Design of variable structure guidance law for head-on interception based on variable coefficient strategy. ActaArmamentarii, 35(1): 134-139. 
[8] Yan L,Zhao J G,Shen H R, et al.2014. Three-dimensional united biased proportional navigation guidance law for interception of targets with angular constraints. ActaAeronauticaetAstronauticaSinica,35(7):1999-2010.

[9] Tyan F,Jeng F S. 2008. Capture region of three dimensional PPN guidance law against a high speed-nonmaneuvering target. Seattle, WA, United States: Institute of Electrical and Electronics Engineers Inc., pp: 3488-3493.

[10] Der-Ren Taur. 2003.Composite guidance and navigation strategy for a SAM against high-speed target. AIAA Guidance, Navigation, and Control Conference and Exhibit.

[11] YuanLiying,Li Jie,Li Shiyong. 2009. Adaptive terminal guidance law based on feedback linearization for maneuvering targets. Journal of Beijing Institute of Technology. (5): 386-389.

[12] Tian Yuan,Ren Zhang.2009. Design of guidance law for exoatmospheric interceptor during itsterminal course. Journal of Astronautics. (2): 474-480.

[13] PengJianliang, Sun Xiuxia, Dong Wenhan. 2009. Construction of augmented proportion navigation law with tracking differentiator. Journal of Applied Science. (4): 435-440.

[14] Li K, Chen L, Tang G. 2013. Improved differential geometric guidance commands for endoatmospheric interception of high-speed targets. Science China: Technological Sciences, 56(2): 518-528.

[15] LeiHumin, Zhang Xu, LiJiong, Liu Tao.2015. Guidance law based on dynamic terminal sliding mode for high speed and maneuvering target. Solid Rocket Technology. (2): 160-165.

[16] Li Yuan, ZhaoJiguang, Yan Liang, Bai Guoyu. 2015. United proportional navigation law for interception of high-speed maneuvering targets. Journal of Beijing University of Aeronautics and Astronautics. (5): 825-834.

[17] Dhananjay N, Ghose D. 2014. Accurate time-to-go estimation for proportional navigation guidance. Journal of Guidance, Control, and Dynamics. pp: 1-6.

[18] Heller C, Yaesh I, Ben-Asher J Z. 2013. Engineering notes: Optimal setting of the proportional-Navigation gain. Journal of Guidance, Control, and Dynamics, 36(3): 888-891.

[19] Goldan O, Gutman S. 2012.Adjoint stability and miss distance in proportional navigation. 1801 Alexander Bell Drive, Suite 500, Reston, VA 20191-4344, United States: American Institute of Aeronautics and Astronautics Inc.

[20] Li Kebo, Chen Lei, Zhang Yi. 2012. Dimension-reduction method of true proportional navigation guidance law. Journal of National University of Defense Technology. (03): 1-5. 
[21] Yanushevsky R. 2007. Modern Missile Guidance. CRC Press, pp: 12-18.

[22] Feng T. 2012. Capture region of a 3D PPN guidance law for intercepting high-speed targets. Asian Journal of Control, 14(5): 1215-1226. 